\title{
A SIMPLE MODEL FOR OILSPILL CONTAINMENT
}

\author{
SHAUN R. BELWARD ${ }^{1}$ and LAWRENCE K. FORBES ${ }^{2}$
}

(Received 2 March, 1998; revised 3 November, 1998)

\begin{abstract}
This paper develops a simple model for the containment of oil behind a boom in water. The flow of water beneath the oil is assumed two dimensional (horizontal and vertical) and perpendicular to the boom. We look for steady solutions and assume the oil is so viscous that the fluid velocity within the oil is zero. We are able to calculate what shape the oilslick will form and under which circumstances the boom will be successful (that is, no oil escapes under the boom) based on the predicted depth of the slick at the boom.
\end{abstract}

\section{Introduction}

The use of boom structures to contain oil spills has been an area of much study in the engineering community in the last quarter of a century. This is not surprising when one considers the damage that occurs when oil spills from an oil tanker, especially in areas regarded as environmentally sensitive. Work on modelling the containment of low viscosity oils is far enough advanced to be used as a prediction tool for recovery teams, see for example Wilkinson [7]. In this work, equilibrium oilslick thicknesses were calculated for both finite and infinite depth fluids under the assumption of uniform flow beneath the oilslick. It was found in finite depth fluids that oil could be contained if the fluid flow beneath the slick did not have a Froude number (see below) too great and that slick thicknesses would not exceed more than about one tenth the depth of the free stream.

Johnston, Fitzmaurice and Watt [4] report that predictive tools for the containment of highly viscous oils are not so well advanced. These authors propose that high viscosity oil contained by a boom may be assumed to act as a Bingham plastic. Their model for high viscosity oil then proceeds by calculating flow quantities in the water

\footnotetext{
'School of Computer Science, Mathematics and Physics, James Cook University, Townsville QLD 4811, Australia.

${ }^{2}$ School of Mathematics and Physics, The University of Tasmania, Hobart TAS 7001, Australia.

(C) Australian Mathematical Society 2001, Serial-fee code 0334-2700/01
} 
on the oil-water interface so that stresses on the oil may be calculated. The oil is then allowed to "deform" as a plastic, thus redefining the oil-water interface. Flow quantities are again calculated and the procedure iterates until the fluid violates a failure criterion, that is, its depth at the boom is greater than the boom depth itself.

In this paper we propose a very simple model for the containment of highly viscous oils by boom structures. We look for steady (that is, time independent) solutions and assume that there is no movement of particles within the oil. Clearly this is an approximation, and a more accurate model would have to allow for movement within the oil, such as in Johnston, Fitzmaurice and Watt [4] or King and Tuck [5]. Note the latter paper deals with upward airflow supporting liquids on sloped plane surfaces (such as may occur on the windscreen of a car at high speed in rain), but that some aspects of the modelling are similar.

The remainder of the paper is as follows. Section 2 contains the formulation of our model. A key part of this formulation is that it allows the investigation of the need for surface tension effects in the model. In Section 3, we discuss the model's predictions under zero surface tension. Section 4 looks at non-zero surface tension solutions while in Section 5, we analyse the predictions of our model and briefly mention areas for further work.

\section{Formulation}

We consider containing an oilspill in shallow water such as may be required if a tanker hits a reef. The oil is to be contained using a floating barrier and we take the flow of water beneath the oil to be two dimensional and perpendicular to the boom. The water is assumed to be inviscid, incompressible and to flow without rotation; far upstream of the slick the water flows uniformly with speed $U$ and has a constant depth $H$. The oil is assumed to be so viscous that there is no flow within the slick which has a constant cross-sectional area, $A$.

We suppose that the oil and water are in contact with the atmosphere on $y=0$, so that $P(y=0)=0$. Let the interface between the oil and water be given by

$$
y=-h(x) \text { for }-L \leq x \leq 0
$$

so that the slick is contained in an (initially) unknown length $L$ with the boom at $x=0$. Only a fluid interface that is stationary with respect to the boom is considered, so partial derivatives with respect to time are identically zero.

We assume that surface tension acts to coalesce the oil, so that on the oil-water interface

$$
p_{o}=p_{w}+T / R
$$




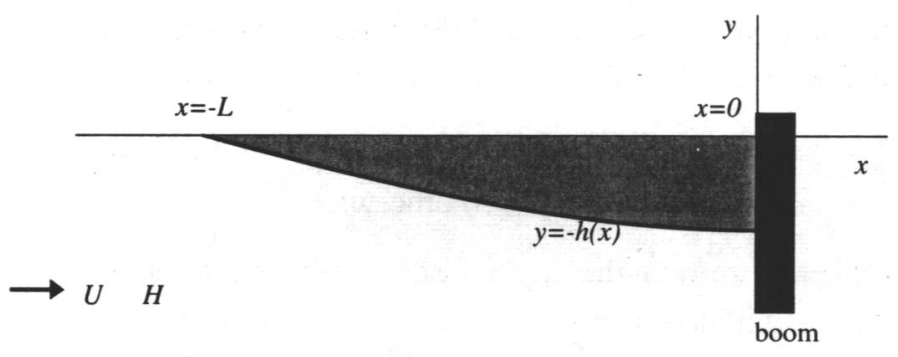

FIGURE 1. A sketch of the fluid system. Note we have exaggerated the vertical scale.

where $p_{o}$ and $p_{w}$ are the pressure in the oil and water respectively, $T$ is the surface tension and $R$ is the radius of curvature of the interface. The density of the water is denoted by $\rho_{w}$ and that of the oil by $\rho_{o}$. Figure 1 shows the fluid system schematically.

The variables above are non-dimensionalised using $H$ as a length scale, $U$ as a velocity scale and $\rho_{w}$ as a density scale. The water then has an upstream uniform speed of 1 and an upstream uniform height of 1 in this nondimensionalised coordinate system. The following dimensionless parameters describing various properties of the flow may now be defined:

$$
\begin{aligned}
G^{2} & =\frac{1-\rho_{o} / \rho_{w}}{U^{2} /(g H)}, & & \text { density-weighted inverse Froude number, } \\
\lambda & =L / H, & & \text { dimensionless length of slick, } \\
\alpha & =A / H^{2}, & & \text { dimensionless area of slick, and } \\
\sigma & =\frac{T}{\rho_{w} H U^{2}}, & & \text { dimensionless surface tension. }
\end{aligned}
$$

The Froude number mentioned in Section 1, defined as $F^{2}=U^{2} /(g H)$, appears frequently in problems of this sort, however in this work it is simpler to use the density-weighted inverse Froude number. Note that $g$ is the acceleration due to gravity. The following work proceeds purely with dimensionless variables.

Since flow is irrotational we may introduce a velocity potential $\phi$, so that the velocity is given by $\mathbf{q}=\nabla \phi$. Continuity then yields

$$
\nabla^{2} \phi=0 .
$$

On the bottom surface the condition of no penetration is

$$
\frac{\partial \phi}{\partial n}=0,
$$


where $\boldsymbol{n}$ is the normal to the surface. We assume that there is no fluid exchange between oil and water, that is, the oil-water interface is a streamline. Thus

$$
\begin{array}{ll}
\partial \phi / \partial n=0 & \text { on } y=-h(x) \text { for }-\lambda \leq x \leq 0 \text { and } \\
\partial \phi / \partial n=0 & \text { on } y=0 \text { otherwise. }
\end{array}
$$

If pressure is taken as zero on the upper surface of the oil, then the Bernoulli equation in the water gives an interface condition

$$
\frac{1}{2}\left(u^{2}+v^{2}\right)+G^{2} y-\sigma \frac{y^{\prime \prime}}{\left(1+\left(y^{\prime}\right)^{2}\right)^{3 / 2}}=\frac{1}{2} \quad \text { on } \quad y=-h(x),
$$

where (1) has been used to obtain the pressure in the water in terms of the pressure in the oil. The $y$ derivatives represent the radius of curvature and $u$ and $v$ are the horizontal and vertical components of the fluid velocity. The remaining condition is obtained from requiring that the oil has constant volume (or in two dimensions, constant cross-sectional area) and is

$$
\int_{-\lambda}^{0} h(x) d x=\alpha
$$

Equations (2) to (7) represent the full nonlinear problem under the assumptions made above. A solution to these equations could be found using a boundary integral technique, such as in Belward and Forbes [1,2] since this solution will satisfy Laplace's equation with several nonlinear boundary conditions. However, our aim in this paper is to present a method which will obtain a quick estimate of how the slick will behave with the boom in place. Therefore we leave the solution of the full nonlinear problem to a later publication. Instead we simplify the problem by using a shallow water approximation.

In using shallow water theory, we assume that the variation of the vertical component of velocity is negligible when compared with the variation of pressure with height, see for example, Stoker [6, page 24]. The horizontal and vertical components of the momentum equation integrate to give

$$
\frac{1}{2} u^{2}-G^{2} h+\sigma \frac{h^{\prime \prime}}{\left(1+\left(h^{\prime}\right)^{2}\right)^{3 / 2}}=\frac{1}{2} \quad \text { for } \quad-\lambda<x<0
$$

and $u=1$ otherwise.

Integrating the continuity equation and using the fact that the interface between oil and water is a streamline gives

$$
u(1-h(x))=1 .
$$

Thus, after using shallow water theory, the problem reduces to solving (8) and (9), subject to the constraint given by (7). Notice that (8) and (9) cannot be treated using a phase-plane analysis, since constraint (7) makes the system non-autonomous. 


\section{Zero surface tension solution}

Undoubtedly, the most difficult aspect of solving (8), (9) and (7) is the complicated derivative term, which represents surface tension effects. In order to obtain quick estimates of slick length, it would be to our advantage if we could ignore this term. In this section we consider this possibility, that is, we look for solutions when $\sigma=0$. It turns out that if this is the case then, if a solution exists, the slick has constant depth so that $h$ is constant.

Under the assumption of zero surface tension (8) becomes

$$
\frac{1}{2} u^{2}-G^{2} h=\frac{1}{2}
$$

The problem is now to solve (9), (10) and (7). Using (9) we eliminate $u$ from (10), obtaining

$$
h\left(1-h / 2-G^{2}\left(1-2 h+h^{2}\right)\right)=0 .
$$

Thus $h=0$ is one solution. Equation (7) then implies that this represents an infinitely long slick, with $\lambda \rightarrow \infty$, so as to conserve the mass of oil. We regard this solution as physically unrealistic, at least for the circumstances we are trying to model here.

Using the quadratic formula on the other factor in (11) gives

$$
h=1-\frac{1}{4 G^{2}} \pm \frac{1}{2} \sqrt{\frac{2}{G^{2}}+\frac{1}{4 G^{4}}} .
$$

The problem then is to determine which of the signs (if any) we can take on the square root. This is achieved by noting that physically realistic solutions will be obtained only when $0<h<1$. It can be shown that this requirement disallows the positive square root, but if $G^{2}>1$ then

$$
h=1-\frac{1}{4 G^{2}}-\frac{1}{2} \sqrt{\frac{2}{G^{2}}+\frac{1}{4 G^{4}}}
$$

is a valid solution. The length of the slick is then determined by the integral condition (7).

If $G^{2}<1$ neither sign in (12) produces a sensible solution. It can be thought that the water is flowing too quickly for the oil to coalesce. In these circumstances the oil will spread into an infinitely long thin film on the top of the water. Perhaps our model falls down here due to the lack of surface tension. If we retain this then recovery may still be possible. 


\section{Non-zero surface tension solution}

In this section we develop the solution to a linearised form of (8), (9) and (7). This is followed by a look at numerical solutions to these equations, generated using a shooting method. The linear solution is obtained by assuming that the area (or in the three dimensional case, volume) of the oilslick is small. That is, we require

$$
\alpha \ll 1 .
$$

Substituting for $u$ from (9) in (8) gives

$$
\frac{1}{2(1-h)^{2}}-G^{2} h+\sigma \frac{h^{\prime \prime}}{\left(1+\left(h^{\prime}\right)^{2}\right)^{3 / 2}}=\frac{1}{2} \text {. }
$$

Now suppose $h(x)=\alpha Y(x)+\mathscr{O}\left(\alpha^{2}\right)$. Substituting this expression into the differential equation (15) and ignoring the contributions $\mathscr{O}\left(\alpha^{2}\right)$ gives

$$
Y^{\prime \prime}+\left(\frac{1-G^{2}}{\sigma}\right) Y=0
$$

Here $\sigma>0$ always, thus the form of the solution to this equation is largely determined by the sign of $1-G^{2}$. If $G^{2}>1$ we get exponential solutions,

$$
Y=\mathscr{A} \exp \left(\sqrt{\frac{G^{2}-1}{\sigma} x}\right)+\mathscr{B} \exp \left(-\sqrt{\frac{G^{2}-1}{\sigma} x}\right) .
$$

There is one boundary condition and one integral constraint,

$$
\begin{aligned}
& Y=0 \text { at } x=-\lambda \text { and } \\
& \int_{-\lambda}^{0} Y(x) d x=1 .
\end{aligned}
$$

Equation (19) expresses the integral constraint (7), accurate to first order in the expansion parameter $\alpha$.

Recall that $\lambda$, the oilslick length in nondimensional coordinates, is a quantity we wish to determine. We may use (18) and (19) to eliminate two of the three unknowns, $\mathscr{A}, \mathscr{B}$ and $\lambda$. This implies that for each pair of the parameters $G$ and $\sigma$ there is a series of solutions, all represented by a hyperbolic sine function. Each solution has a different contact angle on the boom, with the only contact angle not possible being zero, since the hyperbolic sine function cannot satisfy such a condition.

If $G^{2}<1,(16)$ and (18) yield a trigonometric solution,

$$
Y=\mathscr{C} \sin \left(\sqrt{\frac{1-G^{2}}{\sigma}}(x+\lambda)\right) .
$$


The integral condition (19) determines $\mathscr{C}$ or $\lambda$ in terms of the remaining variable.

We note that solutions given by (20) must satisfy $0<\alpha Y(x)<1$ for $-\lambda<x<0$. Since $0<\alpha \ll 1$ this leads to the requirement that

$$
\sin \left(\sqrt{\frac{1-G^{2}}{\sigma}}(x+\lambda)\right)>0 \text { for }-\lambda<x<0 .
$$

This gives the following restriction on $\lambda$ for physically realistic solutions (and hence determines a maximum length for the oilslick) for any choice of the parameters giving sinusoidal solutions,

$$
\lambda<\sqrt{\frac{\sigma}{1-G^{2}} \pi} .
$$

Again for each set of the parameters, there is a series of solutions, each satisfying (19). Each solution has a different contact angle on the boom. Note in this case a contact angle of zero is possible, since the interface profile is sinusoidal.

A solution to the nonlinear equation (15) can be obtained using a shooting method. In this case the variable $x$ is scaled using the unknown oilslick length, $\lambda$, so that $\lambda$ itself appears explicitly in the differential equation. The problem becomes

$$
\frac{1}{2(1-h)^{2}}-G^{2} h+\lambda \sigma \frac{h^{\prime \prime}}{\left(\lambda^{2}+\left(h^{\prime}\right)^{2}\right)^{3 / 2}}=\frac{1}{2}
$$

subject to

$$
\begin{aligned}
\int_{-1}^{0} h(\xi) d \xi & =\frac{\alpha}{\lambda} \\
h(-1) & =0 .
\end{aligned}
$$

For a shooting method to work, we must also specify the contact angle on the boom. Thus we give $h^{\prime}(0)=k$ where $k$ is chosen before computation commences. The algorithm then consists of guessing $\lambda$ and $h^{\prime}(-1)$, and integrating the differential equation, using a Runge-Kutta method. Newton's method is now used to adjust the guess at the two unknowns $\lambda$ and $h^{\prime}(-1)$ so as to make the residuals given by (24) and the condition $h^{\prime}(0)=k$ less than an acceptable numerical tolerance.

\section{Results and discussion}

The calculations performed using zero surface tension $(\sigma=0)$ showed that estimates of oilslick length could be obtained under this assumption only when $G^{2}>1$. It is also noted from (7) and (13) that as the Froude number increases, the depth of the slick will decrease until $G^{2}=1$ when the slick depth is zero, indicating perhaps 


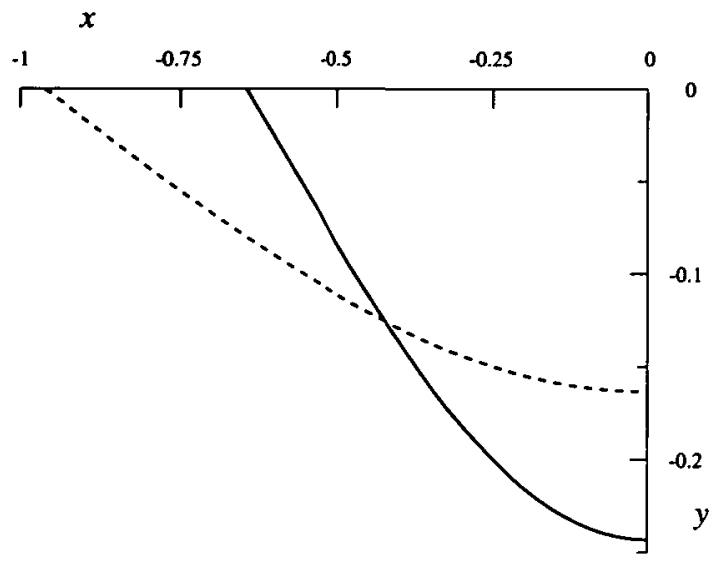

FIGURE 2. Oilslick profiles for the case when $G=0.7454, \sigma=0.1667$ and $\alpha=0.1$. The dashed line represents the linear solution and the solid line the nonlinear solution.

that the slick is an infinite thin film of oil on top of the water and that recovery of oil in these circumstances will be difficult. This is where the $\sigma=0$ assumption fails.

To obtain estimates of oilslick length when $G^{2}<1$ it is necessary to include surface tension effects on the oil-water interface in our model. Under these circumstances, the linear theory gives a solution represented by $(20)$ : If we make the further assumption that the oil-water interface joins the vertical boom at a right-angle, then the slick length is given by

$$
\lambda_{\operatorname{lin}}=\frac{\pi}{2} \sqrt{\frac{\sigma}{1-G^{2}}} .
$$

Investigations of the effects of nonlinearity can be made, as the shooting method mentioned above enables solutions to the nonlinear equations (23) to (25) to be found. In Figure 2 a comparison is made between the linear and nonlinear solutions in a case when $G^{2}<1$. Clearly the linear solution over-estimates the slick length and under-estimates the depth at which the interface between oil and water contacts the boom.

Figure 3 shows the variation of oilslick length with the density-based inverse Froude number for the parameter values $\sigma=0.1667$ and $\alpha=0.1$. The linear solution tends to an infinite length as $G^{2}$ increases to 1 , indicating that recovery of oil may be difficult in these cases. In the nonlinear case this happens at a slightly higher Froude number, however qualitatively the results are the same. Also we note that as $G$ decreases, the slick length decreases substantially and thus that oil is likely to escape under the boom in such circumstances.

Both the linear and nonlinear solutions can be used to study the effects of surface tension on the oilslick. Figure 4 shows how the length of the oilslick varies with 


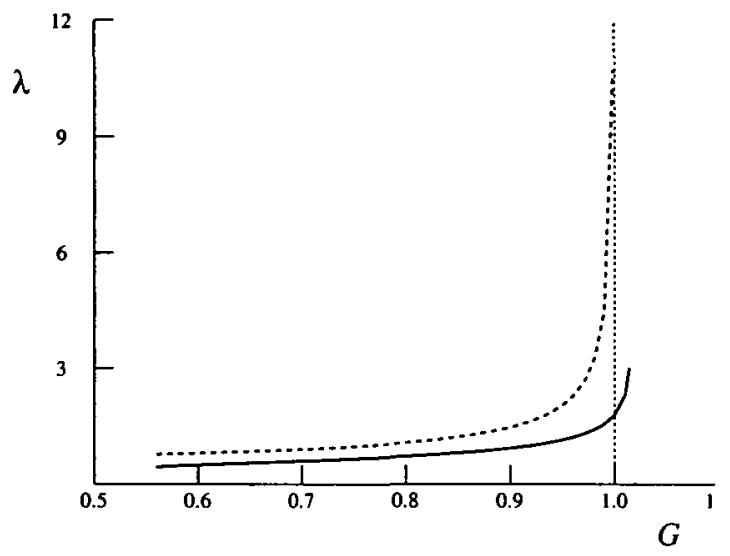

FIGURE 3. Diagram showing how oilslick length varies with Froude number in the case when $\sigma=0.1667$. The dashed line represents the linear theory and the solid line the nonlinear theory. The vertical dotted line represents $G^{2}=1$.

surface tension in the case when $G=0.7454<1$. Both solution types show that the length of the slick decreases as the surface tension decreases and again oil is likely to escape under the boom. Note that this is in contrast to the case where $\sigma=0$ where the theory presented above shows the slick length extending to infinity whenever $G^{2}<1$.

In summary, we have proposed a simple model for the containment of a very viscous oil by a boom structure. It was assumed that a shallow water theory approximation was appropriate within the water, while fluid motion within the oil was taken as zero. It was found that the inclusion of surface tension effects was important in cases when the water was flowing fast enough beneath the oil so that $G^{2}<1$. When $G^{2}>1$, surface tension effects can be ignored, as the model predicts containment of oil in a finite area of water. Note however that when $G^{2} \approx 1$ the solutions should be interpreted as suggesting that containment will be difficult, as the slick will be quite long in either case.

The investigation of the behaviour of the fluid system for cases when $G^{2} \approx 1$ needs to be performed with the more realistic fully nonlinear equations (2) to (7). We comment that attempts were made at finding solutions to (23) to (25) for parameter values satisfying $G^{2}>1$ and $\sigma>0$, however we could not find solutions for any attachment angle $\arctan k$ of the interface with the boom arm. We therefore conclude that the singular nature of the problem is such that, when $\sigma=0$, only solutions for $G^{2}>1$ are possible and that, for $\sigma>0$, solutions can only be obtained with $G^{2}<1$. For each parameter region, singular behaviour is encountered as a limiting configuration, as the value $G^{2}=1$ is approached. An analysis of the exact equations (2) to (7), perhaps using a boundary integral approach, should shed more light on this unexpected behaviour, and will allow a more comprehensive investigation of the fluid 


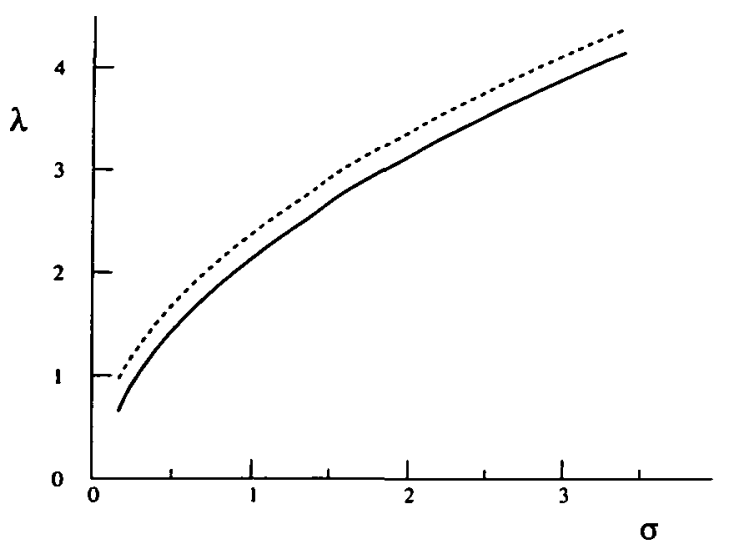

FIGURE 4. Diagram showing how oilslick length varies with surface tension in the case when $G=\mathbf{0 . 7 4 5 4}$. The dashed line represents the linear theory and the solid line the nonlinear theory.

system when $G^{2} \approx 1$. Solving using a boundary integral method will also allow us to generalise the model so that we can study oil spills when the bottom surface of the ocean is not flat or when a shear exists in the velocity profile of the water. Such an approach has already been adopted by Forbes and Belward [3] where a shear was assumed in the velocity profile of air flowing in the atmosphere and the surface of an atmospheric solitary wave was obtained. In the oilslick problem the interface between the oil and the water would be obtained. Studying the possible formation of waves on this interface will be an important step in understanding when and why a boom mechanism for containing oil may fail. We leave this work for a future publication.

\section{References}

[1] S. R. Belward and L. K. Forbes, "Fully non-linear two layer flow over arbitrary topography", J. Eng. Math. 27 (1992) 419-432.

[2] S. R. Belward and L. K. Forbes, "Interfacial waves and hydraulic falls: Some applications to atmospheric flows in the lee of mountains", J. Eng. Math. 29 (1995) 161-179.

[3] L. K. Forbes and S. R. Belward, "Atmospheric solitary waves: some applications to the Morning Glory of the Gulf of Carpentaria", J. Fluid Mech. 321 (1996) 137-155.

[4] A. J. Johnston, M. R. Fitzmaurice and R. G. M. Watt, "Oil spill containment: Viscous oils", in Proceedings of the 1993 Oil Spill Conference, (American Petroleum Institute, Washington, D.C., 1993) 89-94.

[5] A. C. King and E. O. Tuck, "Thin liquid layers supported by steady air-flow surface traction", J. Fluid Mech. 251 (1993) 709-718.

[6] J. J. Stoker, Water waves (Interscience Publishers Inc., New York, 1957).

[7] D. L. Wilkinson, "Dynamics of contained oil slicks", Proc. Am. Soc. Civil Engineers 98 (No. HY6 (hydraulics)) (1972) 1013-1030. 